\title{
OPTIMAL BUFFER ALLOCATION IN TANDEM CLOSED QUEUING NETWORK WITH MULTI SERVERS USING PSO
}

\author{
K.L.Narasimhamu ${ }^{1}$ V.Venugopal Reddy ${ }^{2}$ and C.S.P.Rao ${ }^{3}$ \\ ${ }^{1}$ Department of Mechanical Engineering, AITS, Rajampet, INDIA \\ ${ }_{2}^{2}$ Department of Mechanical Engineering, JNTUCE,Pulivendula, INDIA \\ ${ }^{3}$ Department of Mechanical Engineering, NIT, Warangal, INDIA \\ ${ }^{1} \mathrm{kl}$ simha@gmail.com, ${ }^{2}$ vgreddy7@rediffmail.com
}

\begin{abstract}
Buffer Allocation Problem is an important research issue in manufacturing system design. Objective of this paper is to find optimum buffer allocation for closed queuing network with multi servers at each node. Sum of buffers in closed queuing network is constant. Attempt is made to find optimum number of pallets required to maximize throughput of manufacturing system which has pre specified space for allocating pallets. Expanded Mean Value Analysis is used to evaluate the performance of closed queuing network. Particle Swarm Optimization is used as generative technique to optimize the buffer allocation. Numerical experiments are shown to explain effectiveness of procedure.
\end{abstract}

\section{KEYWORDS}

Buffer Allocation Problem, Closed Queuing Network, Expanded Mean Value Analysis, Particle Swarm Optimization

\section{INTRODUCTION}

Buffer Allocation Problem deals with allocation of optimal buffer slots among intermediate buffer locations of a manufacturing system to achieve a specific objective. The primary reason for having storage buffers is to reduce the idle time because of starving and blocking. Less idle time increases throughput rate of manufacturing system. Buffer needs additional capital investment and floor space. Work-In-Process increases because of buffering in the space available. So total buffer space should be as minimum or with available buffer space total throughput rate should be as maximum as possible. Throughput rate of manufacturing system is a function of service rates of machines and buffer sizes at various machines.

Manufacturing system is combination of machines and queues. Manufacturing system can be shown as network of queues and it can be named as queuing network. A queuing network with constant work-in-process is known as closed queuing network.

David C. Wyld et al. (Eds) : SAI, CDKP, ICAITA, NeCoM, SEAS, CMCA, ASUC, Signal - 2014 


\section{BUfFer Allocation Problem}

BAP is an NP-hard combinatorial optimization problem in design of manufacturing system. In general three types of buffer allocation problem models can be found in the literature.

Model 1: To find optimum buffer allocation in order to maximize throughput rate for a given fixed amount of buffers.

Objective function: Maximize (Throughput rate)

Subject to, Sum of buffers $=$ Total space available.

Model 2: To find optimum buffer allocation in order to minimize total buffer size with desired throughput rate.

Objective function: Minimize (Total buffer size)

Subject to, Throughput rate $\geq$ required (desired) throughput rate.

Model 3: To find optimum buffer allocation in order to minimize Work-in-process inventory with desired throughput rate and total space available.

Objective function: Minimize (Work-in-process inventory)

Subject to, Throughput rate $\geq$ Desired throughput rate

Sum of buffers $\leq$ Total buffer space available.

\subsection{General procedure to solve BAP}

Generative and evaluative methods can be used in cyclic manner to solve BAP as shown in Figure 1.

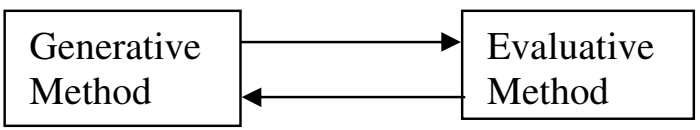

Figure 1. BAP solution process

Evaluative methods are used to obtain the value of objective function. Generative methods are used to search for optimal solution. Evaluative methods can be classified into analytical methods and simulation methods. Further analytical methods can be classified into exact methods and approximate methods. Exact methods are suitable only for small size buffers. Simulation methods are time consuming methods. Generative methods are used to search optimum buffer sizes to optimize system performance. These methods can be classified into traditional and heuristic search algorithms. Sometimes traditional search algorithms cannot jump over local optimum solution in order to find global optimum solution. Meta heuristic methods are strategies to explore search space in order to find optimal or near optimal solutions.

\section{LITERATURE REVIEW}

Daskalaki and Smith [1] attempted BAP, combined with routing in serial parallel queuing networks. An iterative 2-step method was used to solve BAP and routing problem. Expansion method was used as evaluative method and Powell's algorithm was used as generative method. 
Maximization of throughput and minimization of buffer size are the objective functions of this problem.

Smith and Cruz [2] solved BAP for general queuing networks. Minimization of total buffer size is objective function of this problem. The Generalized Expansion Method was used as evaluative method and Powell's algorithm was used as generative technique. Similar work was done by Smith et al. [3] for multi-server queuing networks.

Cruz et al. [4] solved the BAP in an arbitrary queuing networks. Aim was to find minimum buffer size in order to achieve desired throughput. Generalized Expansion Method was used as evaluative method and Lagrangian relaxation method was used as generative method.

Yuzukirmizi et al. [5] considered optimum buffer allocation for closed queuing networks. This is the first procedure to find optimum buffer allocation in closed queuing networks with general topologies and multiple servers. Expanded Mean Value Analysis was used to evaluate throughput of closed queuing network and Powell's algorithm was used to find optimum buffer allocation.

Cruz et al. [6] applied generalized expansion method and multi objective genetic algorithm to optimize throughput and buffer sizes for single server queuing network. Similar work was extended to optimize buffer size, throughput and server rate by Cruz et al. [7].

Soe and Lee [8] presented solutions for tandem queuing networks. Explicit expression was developed as an evaluative method to BAP.

\section{MODEL DESCRIPTION}

In the present work manufacturing system is considered as a closed queuing network with single server (machine) at each node. Buffer size includes the part being operated on machine. All servers are reliable. Maximization of throughput is objective function subjected to sum of buffers is constant.

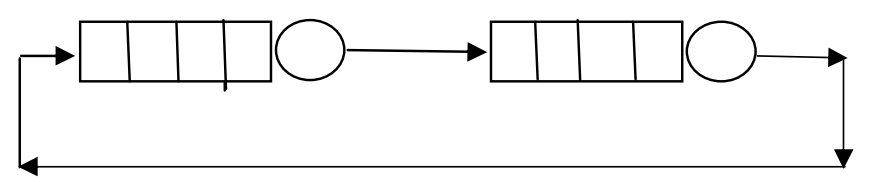

Figure 2.Two node closed queuing network

Two node closed queuing network is shown in Figure 2. Number of components in closed queuing network is constant. Therefore sum of buffers in manufacturing system i.e. work in process of system is constant. Expanded Mean Value Analysis proposed by Yuzukirmizi et al. [5] is used as evaluative method to calculate throughput of manufacturing system which is designed as closed queuing network. Particle Swarm Optimization is used as search method to optimize buffer sizes.

\subsection{Particle Swarm Optimization}

Particle Swarm Optimization (PSO) is one of the metaheuristic search technique to find optimum solution. It is a nature inspired technique. Social sharing of information is main idea behind the implementation of PSO. 
Pseudo code of general PSO algorithm is as follows:

Initialize parameters

Initialize population

Evaluate

Do \{Find pbest (population best)

Find gbest (global best)

Update velocity

Update position

Evaluate

\} While (Termination)

Less complexity and economic computational cost are the main advantages of PSO algorithm.

\subsection{Proposed Algorithm}

Let

M Number of machines (Number of buffers)

B Total buffer space

$\mathrm{N} \quad$ Number of particles

$\mathrm{Mu} \quad$ Service rates of machines

$\mathrm{S} \quad$ Number of servers

$\mathrm{P} \quad$ Number of pallets

Pbest Particle best

Gbest Global best

X Buffer size

$\mathrm{Cp}, \mathrm{Cg}$ Coeffecients (Constants)

Step 1. Generate initial population with n number of particles. Each particle with M number of dimensions.

Step 2. Generate initial velocity matrix (n X M) randomly.

Step 3. Calculate throughput rate for each particle using EMVA algorithm as follows.

Step 3.1. for $\mathrm{P}=1$ to $\mathrm{B}$

Compute throughput rate using $\mathrm{Mu}, \mathrm{S}$

Step 3.2. Find maximum throughput and corresponding number of pallets

Step 4. Find particle best and global best.

Step 5. Calculate change in velocity.

Change in velocity $=\mathrm{Cp} *$ rand ()$*($ Pbest $-\mathrm{X})+\mathrm{Cg} *$ rand ()$*($ Gbest $-\mathrm{X})$.

Step 6 . New velocity $=$ old velocity + change in velocity.

Step 7.Prepare new population as follows.

If new velocity is positive then increase buffer size by one. If new velocity is negative then decrease buffer size by one. Make adjustments to satisfy total buffer size.

Step 8. Assign new velocity to old velocity.

Step 9. If termination condition is satisfied go to step 10. Otherwise go to step3.

Step 10. Finalize the best buffer allocation and total number of pallets. 


\section{NUMERICAL EXPERIMENTS AND RESULTS}

MATLAB code is written to find maximum throughput, optimum buffer allocation and optimum number of pallets in the queuing network. Total buffer size, number of machines, service rates of machines, Number of servers at each node and number of particles are inputs to the program. By executing this program optimum buffer sizes can be obtained. PSO parameters are considered as follows.

Table 1. PSO parameters.

\begin{tabular}{|l|l|}
\hline Parameter & Value \\
\hline Velocity & {$[-4,4]$} \\
\hline $\mathrm{Cp}$ & 0.5 \\
\hline $\mathrm{Cg}$ & 0.5 \\
\hline
\end{tabular}

Experiment 1: Two nodes, $\mathrm{Mu}=[9,1]$, Total buffer size $=6$, Number of servers $=[1,2]$

Two machines are considered with service rates [9,1] and total buffer size 6. Different possibilities are verified for throughput calculation using Expanded Mean Value Analysis. Experiments were conducted and tabulated in table 2. Optimum buffer allocation using proposed algorithm is shown in table 3. It is coinciding with maximum throughput buffer allocation of complete enumeration calculation. Similar experiments with total buffer size 8 and 16 are shown tables from 4 to 9.

Experiment1: Total number of pallets $=6, \mathrm{Mu}=[9$ 1], Servers $=[1,2]$

Table 2. Complete Enumeration for total number of pallets $=6, \mathrm{Mu}=[9$ 1], Servers $=[1,2]$

\begin{tabular}{|c|c|c|c|c|c|}
\hline \multirow{2}{*}{$\begin{array}{l}\text { Number of } \\
\text { pallets }\end{array}$} & \multicolumn{5}{|c|}{ Buffer allocation } \\
\hline & $(1,5)$ & $(2,4)$ & $(3,3)$ & $(4,2)$ & $(5,1)$ \\
\hline 1 & 0.9 & 0.9 & 0.9 & 0.9 & 0.9 \\
\hline 2 & 1.7822 & 1.7822 & 1.7822 & 1.7822 & 1.7822 \\
\hline 3 & 1.946 & 1.9527 & 1.9527 & 1.9527 & 1.9229 \\
\hline 4 & 1.9734 & 1.9881 & 1.9896 & 1.9829 & 1.919 \\
\hline 5 & 1.9779 & 1.9941 & 1.9959 & 1.9816 & 1.91 \\
\hline 6 & 1.9786 & 1.9947 & 1.9948 & 1.9767 & 1.9008 \\
\hline
\end{tabular}

Table 3. Solution using proposed algorithm for total number of pallets=6, $\mathrm{Mu}=[91]$, Servers $=[1,2]$

\begin{tabular}{|c|c|c|}
\hline Optimum Buffer Allocation & Number of Pallets & Maximum Throughput \\
\hline$(3,3)$ & 5 & 1.9959 \\
\hline
\end{tabular}

Experiment 2: Total number of pallets $=8, \mathrm{Mu}=[1,2]$, Servers $[1,2]$ 
Table 4. Complete Enumeration for total number of pallets=8, $\mathrm{Mu}=[1,2]$, Servers $[1,2]$

\begin{tabular}{|l|l|l|l|l|l|l|l|}
\hline \multirow{2}{*}{$\begin{array}{l}\text { Number } \\
\text { of pallets }\end{array}$} & \multicolumn{6}{|l|}{ Buffer allocation } \\
\cline { 2 - 8 } & $(1,7)$ & $(2,6)$ & $(3,5)$ & $(4,4)$ & $(5,3)$ & $(6,2)$ & $(7,1)$ \\
\hline 1 & 0.6667 & 0.6667 & 0.6667 & 0.6667 & 0.6667 & 0.6667 & 0.6667 \\
\hline 2 & 0.9231 & 0.9231 & 0.9231 & 0.9231 & 0.9231 & 0.9231 & 0.9231 \\
\hline 3 & 0.9376 & 0.9811 & 0.9811 & 0.9811 & 0.9811 & 0.9811 & 0.9699 \\
\hline 4 & 0.9349 & 0.9841 & 0.9953 & 0.9953 & 0.9953 & 0.9925 & 0.976 \\
\hline 5 & 0.9317 & 0.9822 & 0.996 & $\mathbf{0 . 9 9 8 8}$ & 0.9981 & 0.9939 & 0.9766 \\
\hline 6 & 0.9289 & 0.9791 & 0.9955 & 0.9988 & 0.9985 & 0.994 & 0.9766 \\
\hline 7 & 0.9265 & 0.9757 & 0.9938 & 0.9984 & 0.9983 & 0.9939 & 0.9766 \\
\hline 8 & 0.9245 & 0.9687 & 0.9915 & 0.9979 & 0.9982 & 0.9939 & 0.9766 \\
\hline
\end{tabular}

Table 5. Solution using proposed algorithm for total number of pallets=8, $\mathrm{Mu}=\left[\begin{array}{ll}1 & 2\end{array}\right]$, Servers $=[1,2]$

\begin{tabular}{|c|c|c|}
\hline Optimum Buffer Allocation & Number of Pallets & Maximum Throughput \\
\hline$(4,4)$ & 6 & 0.9988 \\
\hline
\end{tabular}

Experiment 3: Total number of pallets $=8, \mathrm{Mu}=[2,1]$, Servers $[1,3]$

Table 6. Complete Enumeration for total number of pallets $=8, \mathrm{Mu}=[2,1]$, Servers $[1,3]$

\begin{tabular}{|c|c|c|c|c|c|c|c|}
\hline \multirow{2}{*}{$\begin{array}{l}\text { Number } \\
\text { of } \\
\text { pallets }\end{array}$} & \multicolumn{7}{|c|}{ Buffer allocation } \\
\hline & $(1,7)$ & $(2,6)$ & $(3,5)$ & $(4,4)$ & $(5,3)$ & $(6,2)$ & $(7,1)$ \\
\hline 1 & 0.6667 & 0.6667 & 0.6667 & 0.6667 & 0.6667 & 0.6667 & 0.6667 \\
\hline 2 & 1.2 & 1.2 & 1.2 & 1.2 & 1.2 & 1.2 & 1.2 \\
\hline 3 & 1.4851 & 1.5789 & 1.5789 & 1.5789 & 1.5789 & 1.5789 & 1.5152 \\
\hline 4 & 1.5548 & 1.7042 & 1.7538 & 1.7538 & 1.7538 & 1.7204 & 1.5624 \\
\hline 5 & 1.5663 & 1.7267 & 1.8197 & 1.8483 & 1.8291 & 1.7395 & 1.5373 \\
\hline 6 & 1.5667 & 1.7087 & 1.8279 & 1.8749 & 1.8383 & 1.7095 & 1.5207 \\
\hline 7 & 1.5657 & 1.6826 & 1.7961 & 1.8498 & 1.8044 & 1.6812 & 1.5156 \\
\hline 8 & 1.5651 & 1.6486 & 1.7362 & 1.7971 & 1.7613 & 1.6611 & 1.5149 \\
\hline
\end{tabular}

Table 7. Solution using proposed algorithm for total number of pallets=8, $\mathrm{Mu}=[21]$, Servers $=[1,3]$

\begin{tabular}{|c|c|c|}
\hline $\begin{array}{c}\text { Optimum Buffer } \\
\text { Allocation }\end{array}$ & Number of Pallets & Maximum Throughput \\
\hline$(4,4)$ & 6 & 1.8749 \\
\hline
\end{tabular}

Experiment 4: total number of pallets $=16, \mathrm{Mu}=[0.2,0.5]$, Servers $[5,3]$ 
Table 8. Complete Enumeration for total number of pallets $=16, \mathrm{Mu}=[0.2,0.5]$, Servers $[5,3]$

\begin{tabular}{|l|l|l|l|l|l|l|l|l|}
\hline \multirow{2}{*}{$\begin{array}{l}\text { Number } \\
\text { of } \\
\text { pallets }\end{array}$} & \multicolumn{1}{l}{ Buffer allocation } \\
\cline { 2 - 9 } & $(1,15)$ & $(2,14)$ & $(3,13)$ & $(4,12)$ & $(5,11)$ & $(6,10)$ & $(7,9)$ & $(8,8)$ \\
\hline 1 & 0.1429 & 0.1429 & 0.1429 & 0.1429 & 0.1429 & 0.1429 & 0.1429 & 0.1429 \\
\hline 2 & 0.2857 & 0.2857 & 0.2857 & 0.2857 & 0.2857 & 0.2857 & 0.2857 & 0.2857 \\
\hline 3 & 0.4162 & 0.4286 & 0.4286 & 0.4286 & 0.4286 & 0.4286 & 0.4286 & 0.4286 \\
\hline 4 & 0.5216 & 0.5667 & 0.5702 & 0.5702 & 0.5702 & 0.5702 & 0.5702 & 0.5702 \\
\hline 5 & 0.594 & 0.6912 & 0.7074 & 0.7086 & 0.7086 & 0.7086 & 0.7086 & 0.7086 \\
\hline 6 & 0.6256 & 0.7694 & 0.8135 & 0.82 & 0.8205 & 0.8205 & 0.8205 & 0.8205 \\
\hline 7 & 0.636 & 0.7887 & 0.8703 & 0.8898 & 0.8928 & 0.8931 & 0.8931 & 0.8931 \\
\hline 8 & 0.6449 & 0.7713 & 0.8819 & 0.9222 & 0.9317 & 0.9333 & 0.9335 & 0.9335 \\
\hline 9 & 0.6515 & 0.7484 & 0.8667 & 0.9308 & 0.9512 & 0.9565 & 0.9574 & 0.9575 \\
\hline 10 & 0.6547 & 0.7293 & 0.8392 & 0.9224 & 0.9571 & 0.9687 & 0.9718 & 0.9724 \\
\hline 11 & 0.656 & 0.7173 & 0.8086 & 0.9025 & 0.9522 & 0.9726 & 0.9795 & 0.9812 \\
\hline 12 & 0.6566 & 0.7114 & 0.7821 & 0.8749 & 0.9389 & 0.9695 & 0.9818 & $\mathbf{0 . 9 8 5 4}$ \\
\hline 13 & 0.6568 & 0.7095 & 0.7636 & 0.844 & 0.9177 & 0.9599 & 0.9788 & 0.9852 \\
\hline 14 & 0.6568 & 0.7096 & 0.754 & 0.8148 & 0.8887 & 0.9423 & 0.9702 & 0.9808 \\
\hline 15 & 0.6568 & 0.7104 & 0.7516 & 0.7912 & 0.8531 & 0.9152 & 0.9551 & 0.9726 \\
\hline 16 & 0.6568 & 0.7111 & 0.7532 & 0.7752 & 0.8158 & 0.8792 & 0.9327 & 0.9608 \\
\hline
\end{tabular}

\begin{tabular}{|l|l|l|l|l|l|l|l|l|}
\hline \multirow{2}{*}{$\begin{array}{l}\text { Number } \\
\text { of } \\
\text { pallets }\end{array}$} & \multicolumn{6}{|l|}{ Buffer allocation } \\
\cline { 2 - 9 } & $(9,7)$ & $(10,6)$ & $(11,5)$ & $(12,4)$ & $(13,3)$ & $(14,2)$ & $(15,1)$ & $(9,7)$ \\
\hline 1 & 0.1429 & 0.1429 & 0.1429 & 0.1429 & 0.1429 & 0.1429 & 0.1429 & 0.1429 \\
\hline 2 & 0.2857 & 0.2857 & 0.2857 & 0.2857 & 0.2857 & 0.2857 & 0.2857 & 0.2857 \\
\hline 3 & 0.4286 & 0.4286 & 0.4286 & 0.4286 & 0.4286 & 0.4286 & 0.4235 & 0.4286 \\
\hline 4 & 0.5702 & 0.5702 & 0.5702 & 0.5702 & 0.5702 & 0.5683 & 0.5494 & 0.5702 \\
\hline 5 & 0.7086 & 0.7086 & 0.7086 & 0.7086 & 0.7078 & 0.6996 & 0.6582 & 0.7086 \\
\hline 6 & 0.8205 & 0.8205 & 0.8205 & 0.8201 & 0.8162 & 0.7952 & 0.7281 & 0.8205 \\
\hline 7 & 0.8931 & 0.8931 & 0.8929 & 0.8909 & 0.8799 & 0.8416 & 0.7559 & 0.8931 \\
\hline 8 & 0.9335 & 0.9334 & 0.9322 & 0.9261 & 0.9042 & 0.8504 & 0.76 & 0.9335 \\
\hline 9 & 0.9575 & 0.9568 & 0.9531 & 0.94 & 0.9066 & 0.8449 & 0.7589 & 0.9575 \\
\hline 10 & 0.972 & 0.9697 & 0.9616 & 0.9404 & 0.8989 & 0.8379 & 0.758 & 0.972 \\
\hline 11 & 0.9802 & 0.975 & 0.9615 & 0.9339 & 0.89 & 0.8338 & 0.7577 & 0.9802 \\
\hline 12 & 0.9833 & 0.9748 & 0.9566 & 0.926 & 0.8838 & 0.8325 & 0.7576 & 0.9833 \\
\hline 13 & 0.9824 & 0.9712 & 0.9505 & 0.9196 & 0.8809 & 0.8326 & 0.7576 & 0.9824 \\
\hline 14 & 0.9786 & 0.9663 & 0.945 & 0.9157 & 0.8803 & 0.833 & 0.7577 & 0.9786 \\
\hline 15 & 0.9728 & 0.9613 & 0.9409 & 0.914 & 0.8808 & 0.8333 & 0.7577 & 0.9728 \\
\hline 16 & 0.9659 & 0.9566 & 0.9381 & 0.9136 & 0.8816 & 0.8334 & 0.7577 & 0.9659 \\
\hline
\end{tabular}

Table 9. Solution using proposed algorithm for total number of pallets $=16, \mathrm{Mu}=[0.2,0.5]$, Servers $[5,3]$

\begin{tabular}{|c|c|c|}
\hline $\begin{array}{c}\text { Optimum Buffer } \\
\text { Allocation }\end{array}$ & Number of Pallets & Maximum Throughput \\
\hline$(8,8)$ & 12 & 0.9854 \\
\hline
\end{tabular}

Experiment 5: Total number of pallets $=7, \mathrm{Mu}=[2,0.1,1]$, Servers $[1,2,1]$ 
Table 10. Complete Enumeration for total number of pallets=7, $\mathrm{Mu}=[2,0.1,1]$, Servers $[1,2,1]$

\begin{tabular}{|l|l|l|l|l|l|l|l|l|}
\hline \multirow{2}{*}{$\begin{array}{l}\text { Number } \\
\text { of } \\
\text { pallets }\end{array}$} & \multicolumn{6}{|l|}{ Buffer allocation } \\
\cline { 2 - 9 } & $(1,1,5)$ & $(1,2,4)$ & $(1,3,3)$ & $(1,4,2)$ & $(1,5,1)$ & $(2,1,4)$ & $(2,2,3)$ & $(2,3,2)$ \\
\hline 1 & 0.087 & 0.087 & 0.087 & 0.087 & 0.087 & 0.087 & 0.087 & 0.087 \\
\hline 2 & 0.1723 & 0.1723 & 0.1723 & 0.1723 & 0.1723 & 0.1723 & 0.1723 & 0.1723 \\
\hline 3 & 0.1933 & 0.1938 & 0.1938 & 0.1938 & 0.1934 & 0.1933 & 0.1938 & 0.1938 \\
\hline 4 & 0.1974 & 0.1986 & 0.1987 & 0.1986 & 0.1976 & 0.1974 & 0.1986 & 0.1986 \\
\hline 5 & 0.1982 & 0.1996 & 0.1997 & 0.1995 & 0.1983 & 0.1982 & 0.1996 & 0.1995 \\
\hline 6 & 0.1982 & 0.1998 & $\mathbf{0 . 1 9 9 9}$ & 0.1997 & 0.1984 & 0.1982 & 0.1998 & 0.1996 \\
\hline 7 & 0.1981 & 0.1998 & 0.1999 & 0.1997 & 0.1984 & 0.1981 & 0.1998 & 0.1997 \\
\hline
\end{tabular}

\begin{tabular}{|l|l|l|l|l|l|l|l|}
\hline \multirow{2}{*}{$\begin{array}{l}\text { Number } \\
\text { of pallets }\end{array}$} & \multicolumn{6}{l}{ Buffer allocation } \\
\cline { 2 - 8 } & $(2,4,1)$ & $(3,1,3)$ & $(3,2,2)$ & $(3,3,1)$ & $(4,1,2)$ & $(4,2,1)$ & $(5,1,1)$ \\
\hline 1 & 0.087 & 0.087 & 0.087 & 0.087 & 0.087 & 0.087 & 0.087 \\
\hline 2 & 0.1723 & 0.1723 & 0.1723 & 0.1723 & 0.1723 & 0.1723 & 0.1723 \\
\hline 3 & 0.1934 & 0.1933 & 0.1938 & 0.1934 & 0.1933 & 0.1934 & 0.1929 \\
\hline 4 & 0.1976 & 0.1974 & 0.1986 & 0.1976 & 0.1974 & 0.1976 & 0.1964 \\
\hline 5 & 0.1983 & 0.1982 & 0.1994 & 0.1983 & 0.198 & 0.1982 & 0.1968 \\
\hline 6 & 0.1984 & 0.1982 & 0.1995 & 0.1984 & 0.198 & 0.1983 & 0.1968 \\
\hline 7 & 0.1984 & 0.1981 & 0.1995 & 0.1984 & 0.1979 & 0.1983 & 0.1967 \\
\hline
\end{tabular}

Table 11. Solution using proposed algorithm for total number of pallets=7, $\mathrm{Mu}=[2,0.1,1]$, Servers $[1,2,1]$

\begin{tabular}{|c|c|c|}
\hline $\begin{array}{c}\text { Optimum Buffer } \\
\text { Allocation }\end{array}$ & Number of Pallets & Maximum Throughput \\
\hline$(1,3,3)$ & 7 & 0.1999 \\
\hline
\end{tabular}

Experiment 6: Experiments were conducted for 3 node closed queuing network with various total buffer sizes. Results are shown in table 12.

Table 12. Optimum solutions for 3 node network

\begin{tabular}{|l|l|l|l|l|l|}
\hline $\begin{array}{l}\text { Total } \\
\text { buffer } \\
\text { space }\end{array}$ & Service rates & $\begin{array}{l}\text { Number } \\
\text { of } \\
\text { servers }\end{array}$ & $\begin{array}{l}\text { Optimum } \\
\text { Buffer } \\
\text { Allocation }\end{array}$ & $\begin{array}{l}\text { Optimum } \\
\text { number } \\
\text { of pallets }\end{array}$ & $\begin{array}{l}\text { Maximum } \\
\text { Throughput }\end{array}$ \\
\hline 12 & $(0.3333,1,1)$ & $(3,1,1)$ & $(4,4,4)$ & 10 & 0.7743 \\
\hline 15 & $(0.3333,0.5,0.3333)$ & $(3,2,3)$ & $(5,5,5)$ & 13 & 0.8047 \\
\hline 15 & $(1,1.5,3)$ & $(2,2,1)$ & $(6,6,3)$ & 11 & 1.9269 \\
\hline
\end{tabular}

Experiment 7: Experiments were conducted for 5 node closed queuing network with various total buffer sizes. Results are shown in table 13. 
Table 13. Optimum solutions for 5 node network

\begin{tabular}{|l|l|l|l|l|l|}
\hline $\begin{array}{l}\text { Total } \\
\text { buffer } \\
\text { space }\end{array}$ & Service rates & $\begin{array}{l}\text { Number } \\
\text { of servers }\end{array}$ & $\begin{array}{l}\text { Optimum } \\
\text { Buffer } \\
\text { Allocation }\end{array}$ & $\begin{array}{l}\text { Optimum } \\
\text { number of } \\
\text { pallets }\end{array}$ & $\begin{array}{l}\text { Maximum } \\
\text { Throughput }\end{array}$ \\
\hline 25 & $(0.8,0.8,0.8,0.8,0.8)$ & $(3,2,1,2,3)$ & $(1,1,10,11,2)$ & 20 & 0.7998 \\
\hline 33 & $(4,1,3,2,1.5)$ & $(1,5,2,2,3)$ & $(8,9,3,4,9)$ & 31 & 3.6758 \\
\hline
\end{tabular}

Experiment 8: Experiments were conducted for 8 node closed queuing network with various total buffer sizes. Results are shown in table 14.

Table 14. Optimum solutions for 8 node network

\begin{tabular}{|l|l|l|l|l|l|}
\hline $\begin{array}{l}\text { Total } \\
\text { buffer } \\
\text { space }\end{array}$ & Service rates & $\begin{array}{l}\text { Number of } \\
\text { servers }\end{array}$ & $\begin{array}{l}\text { Optimum Buffer } \\
\text { Allocation }\end{array}$ & $\begin{array}{l}\text { Optimum } \\
\text { number } \\
\text { of pallets }\end{array}$ & $\begin{array}{l}\text { Maximum } \\
\text { Throughput }\end{array}$ \\
\hline 40 & $(1,0.5,1,0.5,1,0.5,1,0.5)$ & $(1,3,1,3,1,3,1,3)$ & $(1,1,5,7,5,7,6,8)$ & 26 & 0.7916 \\
\hline 50 & $(1.2,1, .8,1.2,1,8,1.2,1)$ & $(1,2,3,1,2,3,1,2)$ & $(6,12,1,4,11,2,5,9)$ & 50 & 1.1434 \\
\hline
\end{tabular}

Present work is focused on multi server reliable machines. Work can be extended to solve merge, split, unreliable systems. Extension of this work is under progress by the authors.

\section{REFERENCES}

[1] Daskalaki, S., \& Smith, J. M. (2004). Combining routing and buffer allocation problems in serialparallel queuing networks. Annals of Operations Research, 125, 47-68.

[2] Smith, J. M., \& Cruz, F. R. B. (2005). The buffer allocation problem for general finite buffer queuing networks. IIE Transactions,37(4), 343-365.

[3] Smith, J. M., Cruz, F. R. B., \& Van Woensel, T. (2010). Topological networks design of general, finite, multi-server queueing networks. European Journal of Operational Research, 201(2), 427-441.

[4] Cruz, F. R. B., Duarte, A. R., \& Van Woensel, T. (2008). Buffer Allocation in general single-server queuing networks. Computers, Operations Research 35(11), 3581-3598.

[5] Yuzukirmizi, M., \& Smith, J. M. (2008). Optimal buffer allocation in finite closed networks with multiple servers. Computers, Operations Research, 35, 2579-2598.

[6] Cruz, F. R. B., Van Woensel, T., \& Smith, J. M. (2010). Buffer and throughput trade-offs in M/G/1/K queuing networks: A bicriteria approach. International Journal of Production Economics, 125, 224234.

[7] Cruz, F. R. B., Kendall, G., While, L., Duarte, A. R., Brito, N.L.C.(2012). Throughput maximization of queueing networks with simultaneous minimization of service rates and buffers. Mathematical Problems in Engineering. Volume 2012, Article ID 692593.

[8] Seo, D-W., \& Lee, H. (2011). Stationary waiting times in m-node tandem queues with production blocking. IEEE Transactions on Automatic Control, 56(4), 958-961. 